\title{
UTILIZACIÓN DE TORTA DE CASTAÑA (BERTHOLLETIA EXCELSA) EN LA ALIMENTAGIÓN DE GAMITANA (COLOSSOMA MACROPOMUM)
}

\author{
Gilbert Alagón Hualtpa, Elmer Quispe, \\ Pedro Eufracio, Fidel Pomiano
}

\section{RESUMEN}

El objetivo del estudio fue evaluar la inclusión de torta de castaña $(0,10,20,30$ y $40 \%$ ) en raciones isoenergéticas $\left(2.7 \mathrm{Kcal} \mathrm{g}^{-1} \mathrm{ED}\right)$ e isoproteicas $(25 \% \mathrm{PC})$ y su efecto en el desempeño de gamitana. Se empleó 1000 alevinos $(2,43 \pm 0,37 \mathrm{~g})$ distribuidos en 5 tratamientos en un Diseño Completamente al Azar, alimentados con una tasa inicial de $8 \%$ y final de $1,8 \%$, durante 183 días. Las variables evaluadas fueron afectadas significativamente $(\mathrm{P}<0.05)$ por la variación de los niveles de harina de castaña en las raciones. Los peces alimentados con raciones de $30 \%$ de harina de castaña obtuvieron mejores pesos vivos, mayores ganancias de peso diarias, mayores longitudes totales y mejores índices de conversión alimenticia. El nivel óptimo de harina de castaña establecido fue de $29,26 \%$. El mayor beneficio neto parcial por pescado se presentó a $30 \%$ de inclusión de harina de castaña. Estos resultados revelan que la harina de castaña es una materia prima nutricional y económicamente importante en la región amazónica como fuente de proteína para la alimentación de gamitana.

Palabras clave: Colossoma macropomum, Bertholletia excelsa, nutrición animal, piscicultura, Amazonia.

\begin{abstract}
AbSTRaGt
The objective of this work was to evaluate the inclusion of Brazil nut meal $(0,10$, 20,30 and $40 \%$ ) in portions isoenergetics and isoproteics and its effect in the gamitana acting. 1000 alevinos was used $(2,43 \pm 0,37 \mathrm{~g})$ under a Design Totally at random, fed with an initial and final rate of 8 and $1,8 \%$, in a respective way, during 183 days. The fish fed with portions of $30 \%$ of Brazil nut meal showed bigger weight I live, gain of weight newspaper, longitude total and smaller index of nutritious conversion, being the polynomial pattern that was adjusted to the curve of weight lives and levels of Brazil nut meal of whose equation is derived a good level of $29,26 \% \mathrm{v}$. The biggest partial net profit for fish was presented to $30 \%$ of inclusion of Brazil nut meal. These results reveal the importance zoothecnic and economic of the Brazil nut meal in the gamitana feeding. The level of $30 \%$ of Brazil nut meal in the portion is adapted, guaranteeing good gain of weight and bigger economic retribution.
\end{abstract}

Key words: Colossoma macropomum, Bertholletia excelsa, animal nutrition, fish culture, amazon. 


\section{INTRODUCGIÓN}

La gamitana (Colossoma macropomum), es un pez originario de los ríos Amazonas y Orinoco y sus tributarios (Araujo-Lima y Goulding, 1997). Su dieta natural incluye zooplancton, frutos y semillas, siendo considerado omnívoro con tendencia a frugívoro (Honda, 1974). Es una especie de gran potencial para su crianza en América Latina (Saint-Paul, 1991) debido a su tolerancia a altas temperaturas y bajos niveles de oxígeno disuelto (Alcántara y Guerra, 1990), excelentes cualidades zootécnicas, facilidad de manejo (Graef, 1995), rápido crecimiento (Woynarovich y Woynarovich, 1998), considerándose su importancia desde el punto de vista de seguridad alimentaria e ingreso monetario para la población humana amazónica (Alcántara y Colace, 2001)

En la región Madre de Dios, la actividad castañera representa una importante fuente de ingresos para la población (CMPDAC-MDD, 1999). La recolección de las nueces se efectúa sin alterar fuertemente el bosque, considerándose esta actividad como compatible con la conservación, donde los beneficios económicos dependen de un bosque prístino (Cornejo, s.fi; GP-CFV; 2005; Rumiz, 1999). El subproducto de la extracción de aceite de castaña es una harina de alto tenor proteico, rica en aminoácidos azufrados y buena digestibilidad (Nagashiro et al, 2001; Souza y Menezes, 2004), considerándose su aprovechamiento en la alimentación animal (Ledo, 1996; Nagashiro et al., 2001; Ramos y Bora, 2004), así como en el enriquecimiento de alimentos destinado a la dieta humana (Souza y Menezes, 2004).

Por otra parte, las tendencias actuales en la formulación y fabricación de alimentos para peces es la disminución de costos por kilogramo de alimento producido, debido principalmente a que los peces dependen en gran medida del contenido proteico en la dieta, por lo cual se utilizan ingredientes ricos en ese nutriente (Martínez et al., 1996) que abarcan del 50 al 70\% de la fórmula del alimento (Akiyama, 1992), siendo necesario implementar nuevas fuentes de proteína no convencionales, de bajo costo y que redunden beneficiosamente en los precios del alimento balanceado, aspectos que pueden ser cubiertos en los procesos productivos con el empleo de ingredientes como la harina de castaña.

En este contexto, se efectuó el presente estudio con el objetivo de evaluar el efecto de la inclusión de harina de castaña en la alimentación de gamitana, y contribuir con información que permita disminuir el costo en la elaboración de raciones balanceadas en los sistemas de producción acuícolas de la amazonía peruana.

\section{Materiales Y MÉTODOS}

El experimento fue realizado en Puerto Maldonado, en el Centro de Acuicultura $\mathrm{La}$ Cachuela (GALC), del Fondo Nacional de Desarrollo Pesquero (FONDEPES), durante 183 días. Se empleó un estanque de $1800 \mathrm{~m}^{2}$ de espejo de agua, subdivido en 5 compartimentos o parcelas experimentales, alineados longitudinalmente.

Los alevinos fueron proporcionados por el CALC, con peso promedio de 2,43+0,37 g, distribuidos aleatoriamente en las parcelas a razón de 200 alevinos por tratamiento.

Cinco raciones experimentales fueron formuladas por programación lineal conteniendo $0,10,20,30$ y $40 \%$ de harina de castaña, que representaron los tratamientos A, B, C, D y E, respectivamente (Tabla 1). Los peces fueron alimentados con una tasa inicial de $8 \%$ y $1,8 \%$ al final del experimento.

Tabla 1. Composición porcentual, nutricional y precio de las raciones experimentales por kilogramo (Base húmeda).

\begin{tabular}{lrrrrr} 
& \multicolumn{5}{c}{ Tratamientos } \\
\cline { 2 - 6 } Ingredientes & A & B & C & D & E \\
\hline Harina de maíz & 35,75 & 35,47 & 35,20 & 34,92 & 33,31 \\
Harina de soya & 40,80 & 33,01 & 25,22 & 17,43 & 9,88 \\
Harina de castaña & 0,00 & 10,00 & 20,00 & 30,00 & 40,00 \\
Harina de pescado & 5,00 & 5,00 & 5,00 & 5,00 & 5,00 \\
Aceite de castaña & 7,85 & 5,99 & 4,13 & 2,27 & 1,50 \\
Polvillo de arroz & 7,50 & 7,50 & 7,50 & 7,50 & 7,50 \\
Carbonato de calcio & 1,45 & 1,46 & 1,47 & 1,48 & 1,50 \\
Fosfato monodicálcico & 0,95 & 0,87 & 0,78 & 0,70 & 0,61 \\
Sal & 0,50 & 0,50 & 0,50 & 0,50 & 0,50 \\
Pre mezcla & 0,20 & 0,20 & 0,20 & 0,20 & 0,20 \\
\hline
\end{tabular}

\begin{tabular}{lrrrrr}
\hline Nutrientes (\%, Mcal) & & & & \\
\hline Materia seca & 89,61 & 89,71 & 89,81 & 89,90 & 90,13 \\
Energía digestible & 2,70 & 2,70 & 2,70 & 2,70 & 2,70 \\
Proteína cruda & 25,00 & 25,00 & 25,00 & 25,00 & 25,00 \\
Extracto etéreo & 10,56 & 10,71 & 10,86 & 11,01 & 12,14 \\
Fibra cruda & 4,57 & 4,61 & 4,64 & 4,68 & 4,71 \\
Calcio & 1,00 & 1,00 & 1,00 & 1,00 & 1,00 \\
Fósforo disponible & 0,50 & 0,50 & 0,50 & 0,50 & 0,50 \\
\hline Precio, S/. & 1,46 & 1,32 & 1,18 & 1,04 & 0,93 \\
\hline
\end{tabular}


Al final del experimento se efectuó una pesca que representó un $25 \%$ de la población por tratamiento, midiéndose peso vivo y longitud total a la unidad inferior más cercana de la lectura (Sparre y Venema, 1997).

Los resultados obtenidos fueron sometidos a una prueba de homogeneidad de varianza (Prueba de Levene) y determinar la transformación de datos no-paramétricos. Los valores para peso vivo, ganancia de peso diario, longitud total y conversión alimenticia fueron sometidos a un análisis de varianza de una vía y posteriormente a un análisis por Polinomios ortogonales y determinar la curva de tendencia de las variables de respuesta y niveles de harina de castaña. Los datos fueron procesados con el paquete estadístico SAS (SAS Institute 1990, Cary, NC, USA).

\section{RESULTADOS Y DISCUSIÓN}

El desempeño productivo de los peces frente a los diferentes niveles de harina de castaña en las raciones son presentados en la tabla 2. El análisis de varianza presentó diferencias estadísticas $(\mathbf{P}<0,05)$ para una creciente utilización de harina de castaña como componente de las raciones para gamitana, sobre las variables de respuesta analizadas.

\section{Peso vivo total (G/PeZ)}

La mayor ganancia en peso total se registró en el tratamiento $\mathrm{D}$ con un peso vivo promedio de $786 \pm 208,01 \mathrm{~g}$ y el menor en el tratamiento A con un peso de $610 \pm 135,90 \mathrm{~g}$. Los menores rangos se observan en los tratamientos A y C. Los valores mínimos corresponden a los tratamientos $\mathrm{A}, \mathrm{B}$ y C con $350,00 \mathrm{~g}$, mientras que los máximos a los tratamientos D y E con 1300,00 y $1400,00 \mathrm{~g}$,

Tábla 2. Resultado de las medias de desempeño productivo, Peso vivo (PV), Ganancia de peso diario (GPD), Longitud total (LT), Conversión alimenticia (CA).

\begin{tabular}{|c|c|c|c|c|}
\hline \multirow[b]{2}{*}{ Tratamientos } & \multicolumn{4}{|c|}{ Medias } \\
\hline & s PV (g) & GPD (g) & $\mathrm{LT}(\mathrm{cm})$ & CA \\
\hline$A 0 \%$ & $\begin{array}{r}610,0 \pm \\
135,90\end{array}$ & $3,33+0,74$ & $29,47+2,47$ & $1,43 \pm 0,33$ \\
\hline B $10 \%$ & $\begin{array}{r}691,0 \pm \\
162,79\end{array}$ & $3,76+0,89$ & $31,23+2,20$ & $1,27 \pm 0,33$ \\
\hline C $20 \%$ & $\begin{array}{r}686,0 \pm \\
155,52\end{array}$ & $3,75 \pm 0,85$ & $30,98 \pm 2,29$ & $1,28 \pm 0,34$ \\
\hline D $30 \%$ & $\begin{array}{r}786,0 \pm \\
208,01\end{array}$ & $4,29 \pm 1,14$ & $32,32 \pm 3,03$ & $1,12 \pm 0,28$ \\
\hline $\mathrm{E} 40 \%$ & $\begin{array}{r}706,0+ \\
171,32\end{array}$ & $3,86 \pm 0,94$ & $31,58 \pm 2,60$ & $1,24 \pm 0,28$ \\
\hline
\end{tabular}

respectivamente. La inclusión de mayores niveles de harina de castaña en las raciones experimentales tuvo un efecto significativo $(\mathbf{P}<0,05)$ sobre el peso vivo final.

Bajo similares condiciones (estanques), Arbeláez-Rojas et al. (2002) reportó pesos de 883 g para 170 días de ensayo con pesos iniciales de $90,4 \mathrm{~g}$, alimentados con raciones de $30 \% \mathrm{PC}$; de 673,2 g alimentados con raciones que contenían $24,69 \%$ PC durante 180 días y pesos iniciales de 8,13 g (Padilla, 2000); de $570 \mathrm{~g}$ alimentados con raciones basados en ensilado y harina de pescado durante 120 días y pesos iniciales de $198 \mathrm{~g}$ (Padilla et al., 2000).

El nivel óptimo de harina de castaña para el peso vivo final (Figura 1) se obtuvo a partir del modelo cuadrático, con un valor de $29,26 \%$ de harina de castaña que incrementa hasta $740,09 \mathrm{~g}$ de peso vivo por pez, con un intervalo de confianza al $95 \%$ de 619,89 a $860,27 \mathrm{~g}$ al final de los seis meses de ensayo.

\section{Ganancia de peso diario ( $\mathrm{g} / \mathrm{pez}$ )}

Las mayores ganancias de peso diario (Tabla 2) se observan en las raciones sometidas a efectos de los tratamientos D y E con valores de 4,30 \pm 0,85 y $3,86 \pm 0,96 \mathrm{~g} / \mathrm{pez}$, respectivamente. El menor valor observado para esta variable fue en el tratamiento A (1,91 $\pm 0,74 \mathrm{~g} / \mathrm{pez})$, donde la harina de castaña no fue incluida en la ración experimental. Asimismo, el tratamiento D presenta valores mínimos superiores $(2,46 \pm 1,14$ $\mathrm{g} / \mathrm{pez}$ ) a los demás tratamientos, y el tratamiento E presenta el valor máximo $(7,65 \pm 0,94 \mathrm{~g} / \mathrm{pez})$ para ganancia de peso diario. El rango de peso más pronunciado puede observarse bajo efecto del

Figura 1. Efecto de los niveles de inclusión de harina de castaña sobre el peso promedio final de gamitana.

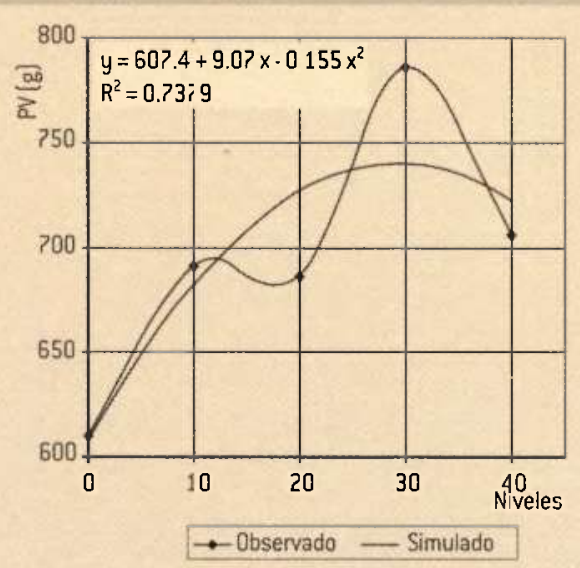


tratamiento $\mathrm{E}$ y el menor en los tratamientos $\mathrm{A}$ y $\mathrm{C}$ con 3,55 g/pez. Respecto al promedio total se reporta $3,80 \pm 0,96 \mathrm{~g} / \mathrm{pez}$ con valores mínimo y máximo de 1,91 y $7,65 \mathrm{~g} / \mathrm{pez}$, respectivamente.

Silva et al. (1996), trabajando con raciones experimentales de sustitución de Pennisetum americanum por žea maíz con $25 \%$ de proteína, durante 189 días, obtuvieron GPD de 2,98; 2,$84 ; 3,34$ y $3,24 \mathrm{~g} / \mathrm{pez}$ para sustituciones de 0 , 20,40 y $60 \%$, respectivamente. GPD de 2,65; 1,8 y $4,5 \mathrm{~g} / \mathrm{pez}$ son reportados por Padilla, $e t$ al. (2000), Padilla (2000) y Arbeláez-Rojas et al. (2002), respectivamente. Las diferencias en GPD reportado por Silva et al. (1996), respecto al presente estudio $(4,30 \mathrm{~g} / \mathrm{pez})$ pudo deberse al peso inicial de los peces, al ajuste energético (EM aves) y a la superficie de los estanques.

El modelo cuadrático es el que mejor ajusta los valores de GPD, cuya derivada de ler. orden arroja el nivel óptimo de inclusión de harina de castaña en las raciones de gamitana, cuyo valor es de $31 \%$ (Figura 2).

\section{Longitud total $(\mathrm{cm})$}

Los peces alimentados con el tratamiento D presentaron el mejor promedio de crecimiento con una longitud total de $32,32 \pm 3,03 \mathrm{~cm}$, seguido de los tratamientos E, B, C y A, siendo este último el de menor promedio con 29,47 $\pm 2,47 \mathrm{~cm}$ (Tabla 2). Asimismo, los valores mínimo y máximo encontrados fueron de 25,50 y $40,00 \mathrm{~cm}$.

La curva de crecimiento para esta variable en función a los niveles de harina de castaña, se refleja en la Figura 3. Similar al comportamiento de Peso vivo y Ganancia de peso diario, se observa

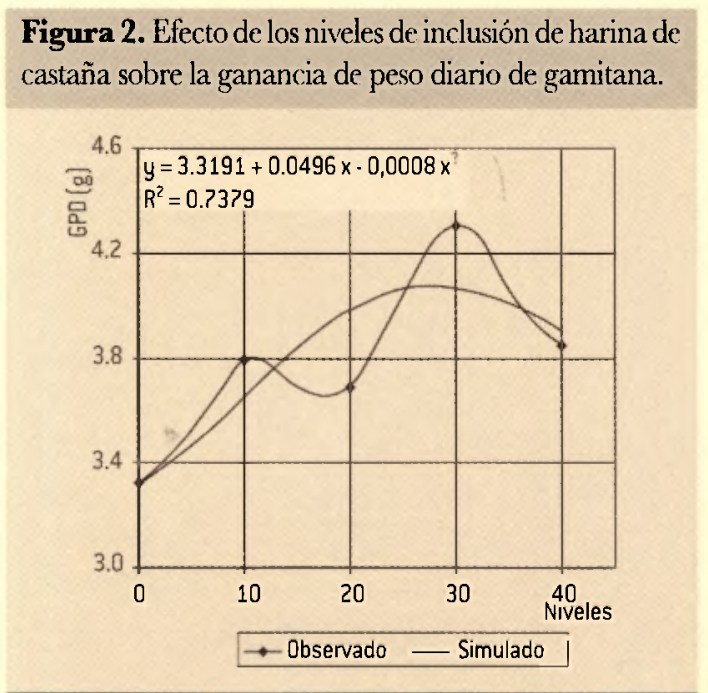

un crecimiento en correlato al nivel de inclusión de harina de castaña hasta un nivel del $30 \%$, luego del cual, se presenta una inflexión que culmina en los valores promedios registrados en el tratamiento $\mathrm{E}$ ( $40 \%$ de inclusión de harina de castaña).

Los resultados de longitud total promedio de gamitana en el periodo de ensayo, son similares a los observados por otros autores que obtuvieron longitudes totales promedio de 26,33 a $33,17 \mathrm{~cm}$, en condiciones experimentales similares (Kohler et al., 2001; Padilla, 2000).

\section{Conversión ALIMENTICIA}

Los valores de conversión alimenticia obtenidos en el presente estudio variaron de 1,12 $\pm 0,28$ a $1,42 \pm 0,33$ para los tratamientos D y A, respectivamente (Tabla 2). El mejor índice de conversión alimenticia se reporta bajo efectos del tratamiento $\mathrm{D}$ con un valor de $1,12 \pm 0,28$. Los tratamientos $\mathrm{B}$ y $\mathrm{C}$, presentan similares índices de conversión, mientras que el tratamiento $\mathrm{A}$ reporta la peor conversión alimenticia, es decir que las gamitanas requieren consumir $1.42 \mathrm{~kg}$ de ración para ganar $1 \mathrm{~kg}$ de peso. El índice de Conversión alimenticia promedio fue de 1,27+0,32.

La curva de Conversión alimenticia en función a los niveles de harina de castaña $y$ ajustado por mínimos cuadrados presenta una relación inversamente proporcional a los niveles de harina de castaña hasta un nivel de $30 \%$ de inclusión en las raciones, según la siguiente figura.

Indices promedio de 3,1 y 2,7 fueron reportados por Padilla et al. (2000) y Padilla (2000), respectivamente. Asimismo, Silva et al. (1996) y Arbeláez-Rojas et al. (2002) obtuvieron índices

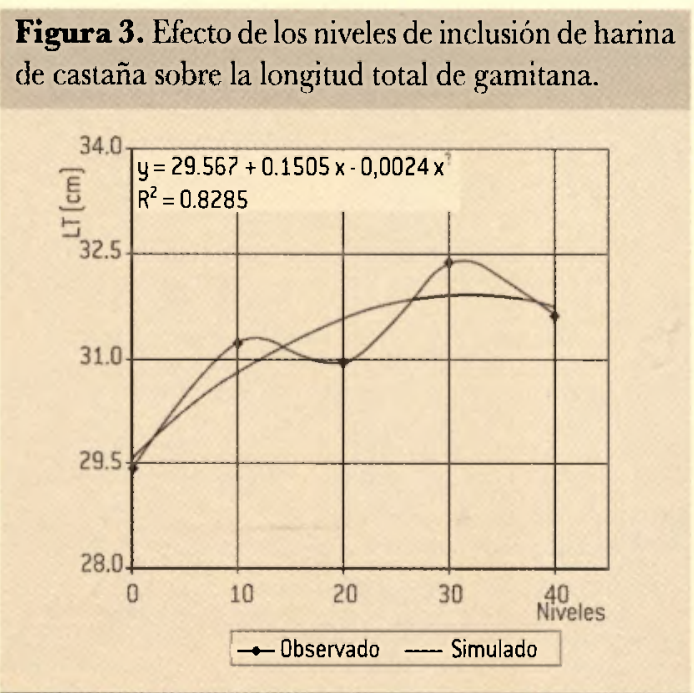


Figura 4. Efecto de los niveles de inclusión de harina de castaña sobre la Conversión alimenticia de gamitana.

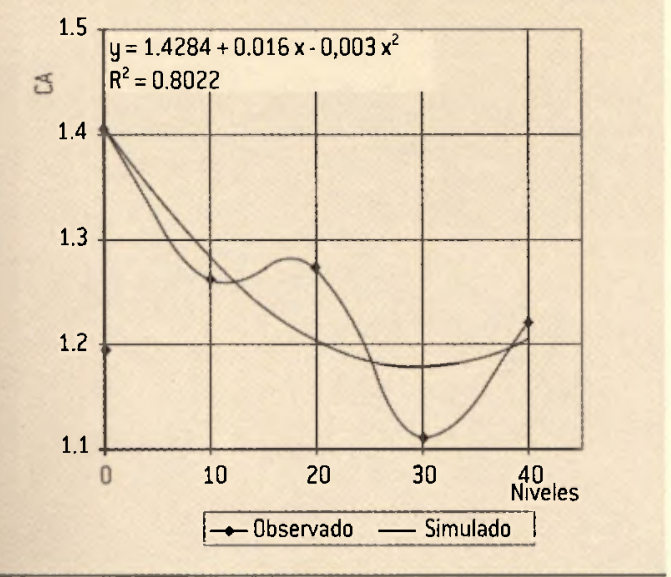

de conversión promedio igual a 2,68 y 1,35 respectivamente.

Bajo diferentes condiciones a las del presente ensayo (estanques), se han logrado índices de conversión de 2,1 (Padilla et al., 1996), de 2,72 (Gutiérrez et al., 1995), de 1,76 (Chagas y Val, 2003). Los índices de conversión alimenticia logrados en el presente experimento $(1,27)$, se consideran mejores comparados con los obtenidos por dichos autores.

La variable por la cual se determinó el nivel óptimo de harina de castaña fue la de Peso vivo al final del experimento (Figura 1). Así, por derivadas, se obtuvo un punto óptimo de $29,26 \%$ de harina de castaña que incrementa hasta 740,09 $\mathrm{g}$ de peso vivo con un intervalo de confianza al $95 \%$ de 619,89 a $860,27 \mathrm{~g}$ al final de los seis meses de ensayo.

El coeficiente de determinación $\mathrm{R}^{2}$ de la ecuación indica que un $74 \%$ del incremento de peso fue debido a los niveles de harina de castaña, el resto fue debido a otras variables concomitantes.

El nivel óptimo de harina de castaña obtenido $(29,26 \%)$, se sitúa dentro del rango del tratamiento D) (30\% de harina de castaña), cuyo efecto produjo el mayor crecimiento de las gamitanas en estudio.

La Tabla 3 presenta el análisis efectuado por la técnica de Presupuestos Parciales. El mejor beneficio neto parcial por pescado se observa bajo efecto del tratamiento $\mathrm{D}(\mathrm{S} / .5,02)$ seguido del tratamiento $\mathrm{E}$ (S/. 4,51).

Los niveles crecientes de harina de castaña influyeron positivamente en el crecimiento de gamitana, sin olvidar que el efecto del alimento
Tabla 3. Análisis de Presupuestos Parciales por tratamientos y pescado.

\begin{tabular}{|c|c|c|c|c|c|}
\hline \multirow[t]{2}{*}{ Componentes } & \multicolumn{5}{|c|}{ Tratamientos } \\
\hline & $\mathbf{A}$ & B & $\mathbf{C}$ & $\mathbf{D}$ & $\mathbf{E}$ \\
\hline \multicolumn{6}{|l|}{ Ingresos } \\
\hline - Ganancia de peso, kg & 0.61 & 0.69 & 0.68 & 0.78 & 0.70 \\
\hline - Precio carne, S/. & 7.5 & 7.5 & 7.5 & 7.5 & 7.5 \\
\hline Beneficio bruto, S/. & 4.56 & 5.16 & 5.13 & 5.88 & 5.28 \\
\hline \multicolumn{6}{|l|}{ Costos variables } \\
\hline - Consumo de alimento, $\mathrm{kg}$ & 0.83 & 0.83 & 0.83 & 0.83 & 0.83 \\
\hline - Costo por kilo de alimento & $0, \mathrm{~S} / .1 .4$ & 461.32 & 1.18 & 1.04 & 0.93 \\
\hline - Costo consumo alimento, & S/.1.21 & 1.09 & 0.97 & 0.86 & 0.77 \\
\hline Total costos variables, $\mathrm{S} /$. & 1.21 & 1.09 & 0.97 & 0.86 & 0.77 \\
\hline \multicolumn{6}{|l|}{ Beneficio neto parcial } \\
\hline - En S/. por pescado & 3.35 & 4.07 & 4.15 & 5.02 & 4.51 \\
\hline $\begin{array}{l}\text { - En porcentaje del } \\
\text { ingreso total }\end{array}$ & 73.547 & $78.88 \varepsilon$ & 80.998 & 85.388 & 85.45 \\
\hline
\end{tabular}

en los peces está condicionado por la tasa de alimentación y por los requerimientos nutricionales. Sin embargo, considerando que el efecto del alimento en el crecimiento de los peces depende del manejo alimentario, tamaño, edad, condición fisiológica, temperatura del agua (Hepher, 1993; Tacon, 1989; Araujo-Lima y Goulding, 1997), se requiere especial atención en la alimentación, ya que representa un $70-80 \%$ de los costos variables de producción (Akiyama,1992).

El desempeño productivo obtenido en esta investigación puede ser atribuido a la calidad proteica de la harina de castaña, rica en aminoácidos azufrados, generalmente de menor tenor en otras fuentes de proteína vegetal (Clement, 1993; Souza y Menezes, 2004). Además puede considerarse la densidad de crianza empleada, debido a que densidades mayores a 0,5 peces $\mathrm{m}-2$ ocasionan bajas producciones, debido al espacio físico, deterioro de la calidad del agua y aglomeración de los peces (Silva-Acuña y Guevara, 2002).

Durante el periodo de ensayo se observó una tasa de sobrevivencia superior al $95 \%$, no obstante el manipuleo a que fueron sometidas las gamitanas en cada muestreo; lo anterior corrobora la resistencia de gamitana, factor importante en su implementación en sistemas de producción acuícolas.

La calidad del agua es uno de los factores determinantes en el desarrollo de la piscicultura, 


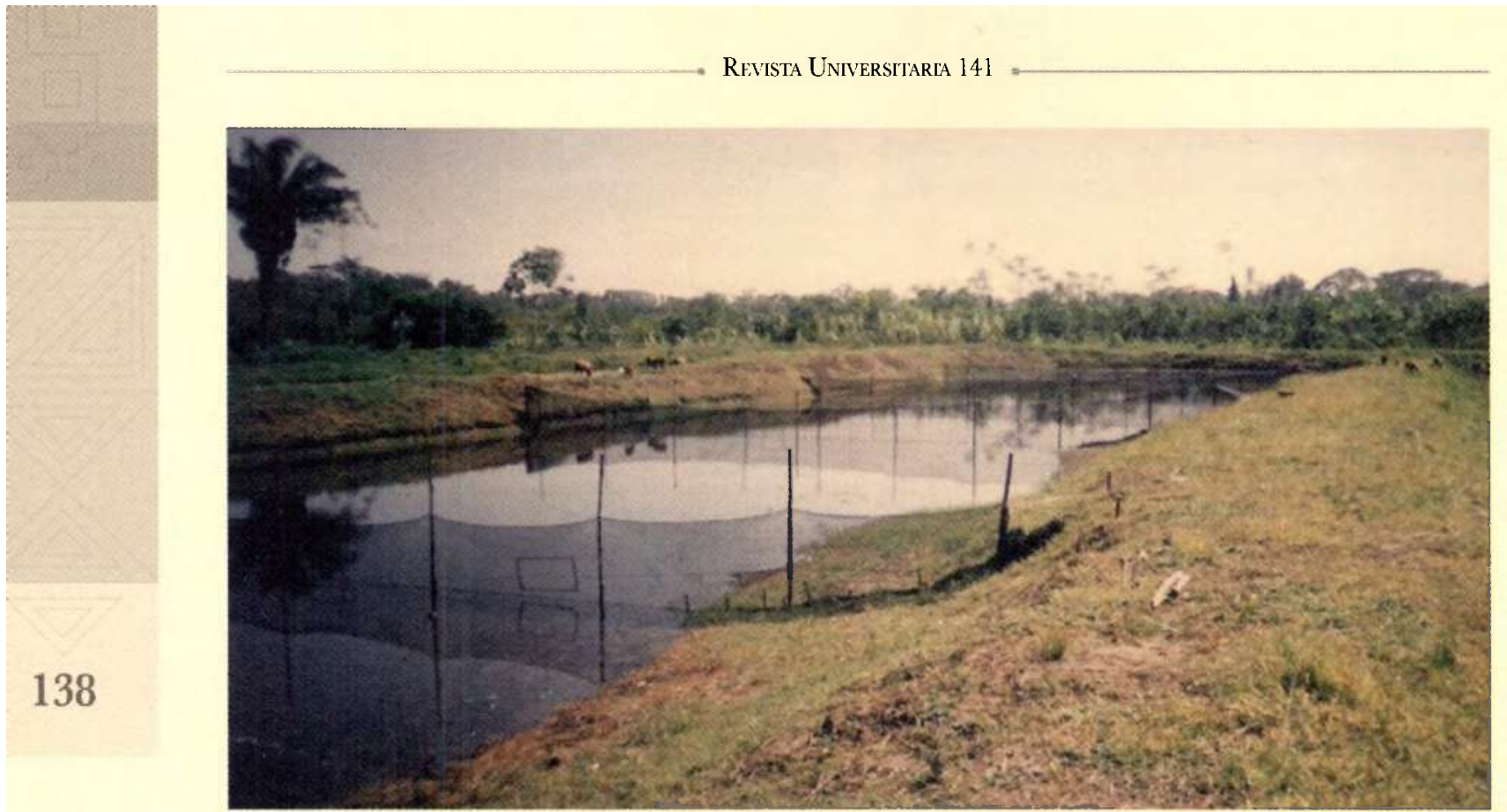

Campo experimental. Espejo de agua: $1800 \mathrm{~m}^{2}$; profundidad promedio: $1-1,20 \mathrm{~m}$

que influye directamente en el crecimiento de los peces. En los poiquilotermos el crecimiento es afectado por la temperatura, presentando correlación positiva hasta un nivel óptimo (Hepher, 1993). Las concentraciones de oxígeno disuelto son muy inconstantes en los cuerpos de agua de áreas inundables durante el curso del año en la amazonía. Los niveles disminuyen a menudo por debajo de $1 \mathrm{mg} \mathrm{l}^{-1}$ durante semanas e incluso meses en algunas áreas. En estas condiciones las gamitanas, juveniles y adultos, muestran adaptaciones morfológicas, fisiológicas y conductas en respuesta a los bajos niveles de oxígeno (AraujoLima y Goulding, 1997).

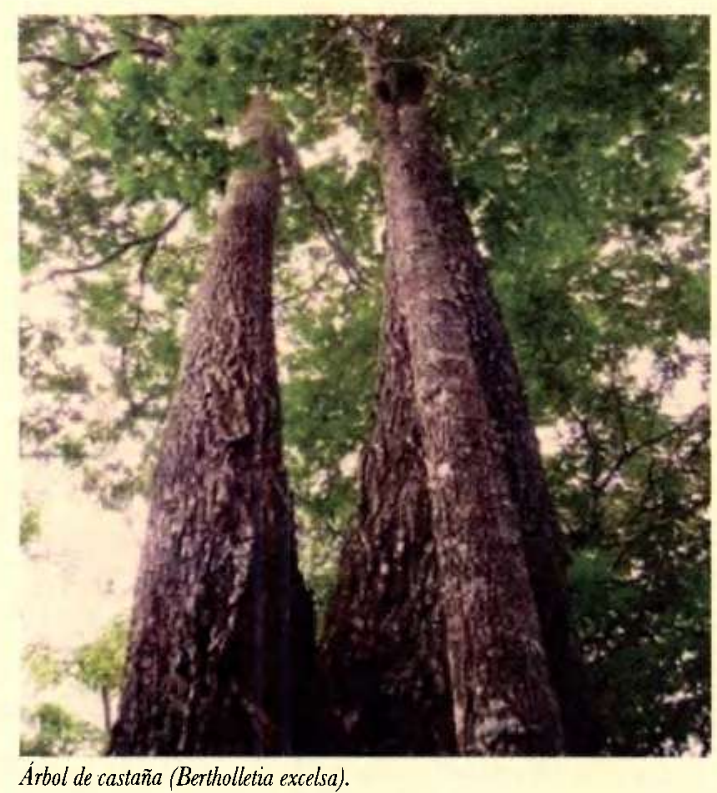

Durante el presente experimento, la temperatura media del agua estuvo en $27,9^{\circ} \mathrm{C}$, considerándose temperatura adecuada para el normal desarrollo de la especie. El nivel promedio de oxígeno disuelto fue de $3,12 \mathrm{mg} \mathrm{l}^{-1}$, considerado dentro del rango aceptado para la crianza de gamitana (Padilla, 2000).

Los resultados obtenidos demuestran la factibilidad de la utilización de harina de castaña como fuente de proteína vegetal en la formulación de raciones en la alimentación de gamitana.

\section{Conclusión}

El estudio demuestra que la harina de castaña es potencialmente útil como fuente primaria de proteína vegetal en la alimentación de gamitana. Se obtuvieron resultados excelentes con un $30 \%$ de harina de castaña como componente de las raciones. Es el nivel recomendado que garantiza buena ganancia de peso y mayor retribución económica.

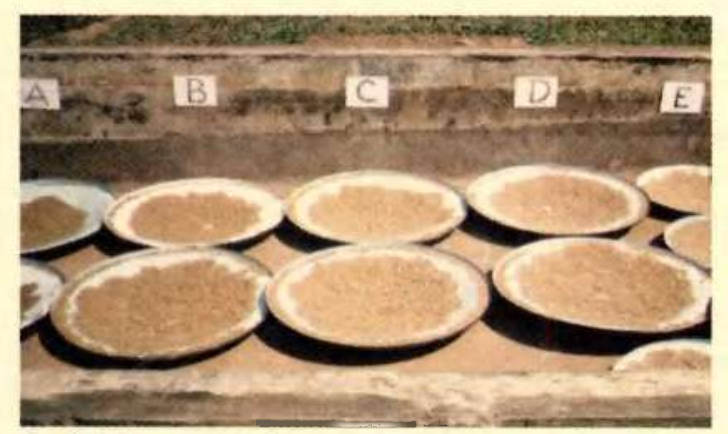

Secado de alimentos paletizados por tratamientos. 


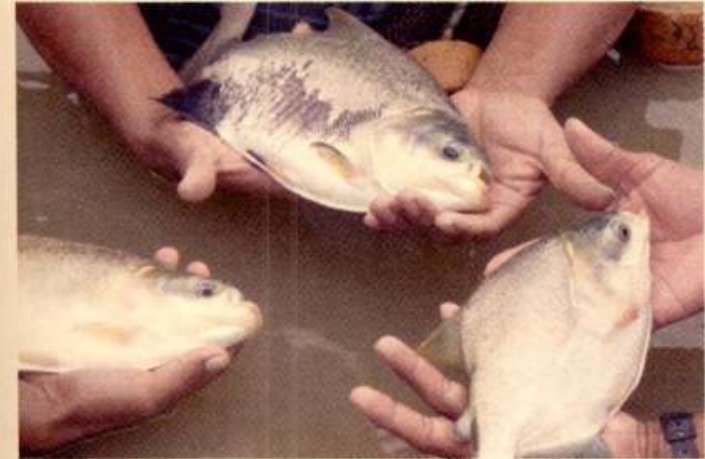

Ejemplares de gamitadas al final del experimento.

\section{BiBLIOGRAFÍA}

\section{AKIYAMA, D.M.}

1992. "Utilización de la pasta de soya en los alimentos acuícolas". In: Soyanoticias Jul-Sep 92 p. 1-8.

\section{ALCÁNTARA, B.F, y COLACE, B.M}

2001. "Piscicultura, seguridad alimentaria y desarrollo sostenible en la carretera Iquitos-Nauta y el río Tigre". PROSEAL UPE II AP-Terra Nuova. 69 pp.

ALCÁNTARA, B. F. y GUERRA, F. H.

1990. "Aspectos de alevinaje de las principales especies nativas utilizadas en piscicultura en la amazonia peruana". In: Folia Amazónica vol. 2 (2) - 1990 p. 139-161.

ARAUJO-LIMA, C. and GOULDING, $M$.

1997. So Fruitul a Fish: Ecology, Conservation, and Aquaculture of the Amazon's Tambaqui. New York, Columbia University Press.

\section{GLEMENT, C.R.}

1993. "Brazil nut". In: Clay,J. W. and Clement, C.R. Selected species and strategies to enhance income generation from Amazonian forest. Misc/93/6 working paper. Rome, FAO.

CMPDAC-MDD (Comisión Multisectorial para la Promoción y Desarrollo de la Actividad Castañera en Madre de Dios).

1999. Plan estratégico de la castaña (Bertholletia excelsa H.B.K.). Prompex, CTAR-MDD.

\section{CORNEJO, $F$.}

s.f. Histona natural de la castaña (Bertholletia excelsa Humb $\mathfrak{E}$ Bonpl.) y propuestas para su manejo. Puerto MaldonadoPerú, Proyecto Conservando Castañales. ACCA.

CP-CFV (Consejo Peruano para la Certificación Forestal Voluntaria). WWF Perú.

2005. Estándar para la certificación del manejo forestal con fines de producción de Castaña (Bertholletia Excelsa) en Peri. CP. CFV, WWF PERÚ-CEDEFOR.

GRAEF, E.W.

1995. As espécies de peixes com potencial para criaçao no Amazonas. In: Val, A. L.; Honczaryk, A. Criando peixes na Amazonia. Manaus: INPA.

HEPHER, B.

1993. Nutrición de peces comerciales en estanques. Edit. Limusa. México. 406p.

HONDA, E. M.

1974. Contribuicãa ao conhecimento da biologia de peixes do Amazonas. II. Alimentação de Tambaquí, Colossama bidens (spix). Acta amazónica. 4(2):47-53.

\section{Agradecimiento}

Los autores agradecen a las estudiantes Haydee López, Leslie Cusi, Janet Alvarez; a los técnicos Alfredo Gonzáles, Leocadio Trejo y al Bach. Renán Meza, por su importante ayuda durante el trabajo de campo.

LEDO, A. da S.

1996. Potencialidade da fruticultura no estado do Acre. Rio Branco, AC: EMBRAPA-CPAF. 16 p. (EMBRAPA-CPAF-AC. Documentos, 20).

MARTÍNEZ, C.A.; CHÁVEZ, M. G.; OLVERA, M. A. y ABDO, M. I.

1996. "Fuentes alternativas de proteínas vegetales como substitutos de la harina de pescado para la alimentación en acuicultura." In: Cruz, L. E; Ricque, D. y Mendoza, R. (Eds.). Avances en Nutrición Acuicola III. Memorias del Tercer Simposium Internacional de Nutrición Acuícola, 11 al 13 de noviembre de 1996. Nuevo León, México, Universidad Autónoma de Nuevo León, Monterrey.

NAGASHIRO, C. W. ; SAUCEDO, A.; ALDERSON, E.; WOOD, G. D. y NAGLER, M. J.

2001. Chemical composition, digestibility and aftatoxin content of Brazil nut (Bertholletia excelsa) cake produced in north-eastern Bolinia. Livestock Research for Rural Development, vl3, n2.

\section{PADILLA, P.P}

2000. "Efecto del contenido proteico y energético de dietas en el crecimiento de alevinos de gamitana (Colossoma macropomum)". En: Folia Amazónica vol. 10 (1-2) p. 81-90.

RAMOS, C. M. P.; Bora, P. S.

2004. "Functional characterization of acetylated Brazil nut (Bertholletia excelsa HBK) kernel globulin." In: Ciênc. Tecnol. Aliment., vol. 24, no. 1, p. 134-138.

RUMIZ, D. I.

1999. "La explotación de recursos no maderables en el norte de Bolivia y su impacto sobre la fauna silvestre". Boletin 17. BOLFOR.

\section{SAINT-PAUL, U.}

1991. "The potential for Colossoma culture in Latin American". In: Info fish International, v. 2, p. 49-53.

\section{SAS Institute, Inc.}

1990. SAS / STAT User's guide, Version 6. 4th edition. SAS Inst. Inc., Cary, NC.

SILVA,J. A. M. DA; PEREIRA-FILHO, M.; OLIVEIRAPEREIRA, M. I.

2000. "Seasonal variation of nutrients and energy in tambaqui's (Colossoma Macropomum Cuvier, 1818) In: NATURAL FOOD. REV. BRAS. BIOL., VOL.60, NO.4, P.599-605. 
SILVA-ACUNA, A. y GUEVARA, $M$.

2002. "Evaluación de dos dietas comerciales sobre el crecimiento del hibrido de Colossoma macropomum x Piaractus brachypomus". En: Zootecnia Tropical, 20(4):449-459.

SOUZA, M. L. DE; MENEZES, H. C. de

2004. "Processamentos de amêndoa e torta de castanha-dobrasil e farinha de mandioca: parâmetros de qualidade". Em: Ciènc. Tecnol. Aliment., vol.24, no.l, p. 120-128.

SPARRE, P. y VENEMA, S. C.

1997. Introdución a la coaluación de recursos pesquecros tropicales.
Parte I. Manual. Documento Técnico de Pesca. $\mathrm{N}^{\circ}$. 306.1 Rev. 2: Roma, FAO.

TACON, A. G. J.

1989. Nutrición y alimentación de peces y camaromes cultivados, Manual. de capacitacion. GCP/RI_A/I02/ITA. Documento de campo $\mathrm{N}^{\circ}$ 4. Brasilia, FAO.

WOYNAROVICH, A. y WOYNAROVICH, E.

1998. Reprodución artifical de las especies Colossoma y Piaractus. Gría detallada para la reprodtción de alerinos de gamitana, paco y caraña. Lima, FONDEPES. 